\title{
Niveles de elementos traza en tejidos cardiovasculares de pacientes sometidos a cirugía cardíaca en la región de Antofagasta, Chile
}

\author{
Domingo Román ${ }^{1}$, Carlos Solar ${ }^{2}$, Isabel Pizarro ${ }^{3 .}$.
}

\author{
1 Laboratorio de Química Bioinorgánica y Analítica Ambiental. \\ Departamento de Química. Facultad de Ciencias Básicas. Universidad de Antofagasta - Chile \\ 2 Unidad de Cirugía Cardiovascular. Clínica Antofagasta - Chile
}

Introducción: La población de la Región de Antofagasta se encuentra expuesta a contaminación ambiental por metales pesados (MP). Los elementos traza (ET) podrían actuar con As como agonistas o antagonistas. El presente estudio detalla la determinación de las concentraciones totales (CT) de V, Cr, Mn, Co, Ni, Cu, As, Se y Boro en aurícula derecha $(\mathrm{AD})$, arteria mamaria izquierda (AMI), vena safena (VS) y tejido adiposo (TA) de personas que padecen patologías cardiovasculares (CV) y han requerido cirugía cardíaca.

Métodos: Se analizaron AMI, VS, AUD, y TA en un grupo de 50 pacientes con cardiopatía coronaria de la Región de Antofagasta que han sido sometidos a cirugía cardíaca (grupo expuesto, E), y en un grupo de 20 pacientes control sometidos a cirugía cardíaca en el mismo período en Santiago (grupo no expuesto, $\mathrm{NE}$ ). Las determinaciones de ET y MP se realizaron mediante Técnicas Ópticas y Atómicas.

Resultados: En el grupo de pacientes E residentes en la Región de Antofagasta Boro presenta correlaciones interelementales significativas $(p=0,05)$, que no se presentan o son escasas en el grupo NE. El rango de CT de B en AD fue de 24,721 - 932,032 $\mu \mathrm{g} / \mathrm{g}$, niveles muy superiores a los encontrados en el resto de los elementos en estudio. El rango de CT de B encontrada para $\mathrm{AD}$ fue de $25,205-56,873 \mu \mathrm{g} / \mathrm{g}$.

Conclusión: Individualmente, el elemento más enriquecido en todos los tipos de tejidos del grupo $\mathrm{E}$ de la Región de Antofagasta es B, le siguen As y Mn. Cu está empobrecido en el grupo NE, en particular en AD y AMI.

Correspondencia:

Isabel Pizarro

E-mail: ipizarro@uantof.cl 


\section{Trace elements in cardiovascular tissues of patients undergoing cardiac surgery in a heavy metal contaminated area, the Antofagasta region (Chile)}

The Antofagasta population is exposed to environmental contamination with heavy metals (HM). Trace elements (TE) might interact with As either as agonists or antagonists. The total concentracions of $\mathrm{V}, \mathrm{Cr}, \mathrm{Mn}$, $\mathrm{Co}, \mathrm{Ni}, \mathrm{Cu}, \mathrm{Se}$ and $\mathrm{B}$ in the right atrium (RA), left internal mammary artery (LIMA), saphenous vein (SV) and adipose tissue (AT) from patients undergoing cardiac surgery are reported

Methods: LIMA, SV, RA and AT from 50 patients with myocardial infarction submitted to revascularization surgery in Antofagasta (exposed group, E) or Santiago (non exposed group NE, 20 patients) were analyzed. Optical and atomic techniques were used for analysis of HM and TE.
Results: Significant inter elemental correlations were observed for B in group $\mathrm{E},(\mathrm{p}<0.05)$, which are not present in group NE. The RA concentrarion of B in group A ranged from 24.72 to $932.03 \mathrm{ug} / \mathrm{g}$, substancially higher than concentracion of the other elements under study. Corresponding concentrarions of $\mathrm{B}$ in the $\mathrm{NE}$ group were 25.21 to 56.87 $\mathrm{ug} / \mathrm{g}$.

Conclusion: B was the most enriched element in the exposed group, followed by As and Mn. Cu content was significantly lower in RA and LIMA from patients in the non exposed group.

Key words: Environmental contamination, heavy metals, trace elements, cardiovascular tissues.
Introducción: En condiciones de vida saludable, varios metales y metaloides desempeñan roles o funciones bioquímicas inorgánicas esenciales para la vida de la especie humana ${ }^{1,2}$. Esto caracteriza a los denominados elementos traza (ET) y establece una clara diferencia con los denominados metales pesados (MP), aunque estos últimos pueden también adoptar formas químicas biodisponibles. Los ET también presentan propiedades ecotóxicas y efectos adversos cuando los niveles de concentración natural se ven modificados o alterados ${ }^{3}$.

Poco se sabe aún sobre el exacto comportamiento bioquímico inorgánico esencial de los denominados ET a saber, $\mathrm{Cd}, \mathrm{Pb}, \mathrm{As}, \mathrm{Se}, \mathrm{B}$ y otros ${ }^{4}$. Sobre la base de revisiones epidemiológicas y experimentos con animales, los ET han sido clasificados en esenciales, no esenciales y tóxicos. Sin embargo, la frontera entre los esenciales y tóxicos en ciertos casos es extraordinariamente sutil, ya que varios presentan ambos comportamientos ${ }^{5}$, dependiendo del nivel de concentración en el compartimiento biológico del caso, de la concentración medioambiental en el nicho ecológico fuente de suministro del elemento y del patrimonio genético de la especie biológica.

Las relaciones interelementales son un hecho reconocido en el ámbito químico bioinorgánico ${ }^{6}$. As interactúa con otros elementos traza ambiental y biológicamente. Con Se y $\mathrm{Zn}$ la toxicidad inducida por As disminuye, mientras que con $\mathrm{Pb}$ y $\mathrm{Cd}$ la toxicidad puede verse incrementada; $\mathrm{a}$ su vez, As inhibe las enzimas que intervienen en la biosíntesis del grupo hemo causando porfiria ${ }^{7}$. En invertebrados As y Mo exaltan la toxicidad de Se. Sin embargo, se exal- 
ta la toxicidad de As o Mo sólo a niveles relativamente altos de $\mathrm{Se}^{8}$.

A fines de 1975 se descubrió que Se era un elemento esencial irremplazable para los animales y seres humanos. Una década antes se había informado una notable correlación geográfica, en lugares donde el suelo tenía más Se la tasa de mortalidad por cáncer era menor. Por este y otros antecedentes similares, se considera que Se ejerce una importante protección contra agentes carcinogéni$\cos ^{9}$. Sin embargo, entre los micro-nutrientes metálicos esenciales para los humanos Se es el más tóxico debido a que su umbral de concentración entre la deficiencia, status normal y nivel tóxico es muy estrecho ${ }^{10}$. Experimentos en mamíferos han mostrado que Se (IV) puede ejercer un efecto protector contra la toxicidad de MP como Cd, $\mathrm{Pb}, \mathrm{Hg}$ y $\mathrm{As}^{11}$.

Estudios epidemiológicos han mostrado que bajos niveles de Se (IV) en la sangre pueden contribuir al incremento de las enfermedades neoplásicas y $\mathrm{CV}^{12}$. Sin embargo, los mecanismos bioquímicos inorgánicos de esta acción protectora no han sido aún totalmente elucidados, y en el caso de las enfermedades CV son controversiales ${ }^{13}$. Un mecanismo viable podría ser el relacionado con la reducción de los niveles de lipoproteínas de baja densidad (LDL) en la sangre ${ }^{14}$.

La población de la Región de Antofagasta se encuentra ambientalmente expuesta al riesgo de MP. Si bien es cierto que en la Región de Antofagasta el factor ambiental que más se asocia con cáncer es la ingestión y el contacto de As por vía oral y aérea, en el ámbito médico regional existe la fundada presunción que ello también se refleja en otras patologías como las enfermedades CV. En el caso del cáncer como en el de las patologías CV, los ET también podrían actuar cooperativamente con As como agonistas o antagonistas ambientales ${ }^{15}$ o por si solos, convirtiéndose en un factor adverso importante en la calidad de vida de un número importante de personas. De la revisión bibliográfica, se ha podido inferir que los elementos más involucrados en la salud $\mathrm{CV}$ humana serían $\mathrm{Ca}$, $\mathrm{Mg}, \mathrm{V}, \mathrm{Mn}, \mathrm{Cr}, \mathrm{Ni}, \mathrm{Co}, \mathrm{Cu}, \mathrm{Zn}$, As y $\mathrm{Se}$, pero por razones geomédicas, $\mathrm{B}$ también ha sido incluido ${ }^{16}$. El presente estudio detalla la distribución de las concentraciones totales de los elementos antes mencionados en AD, AMI, VS y TA de personas que han presentado un infarto del miocardio y han requerido cirugía cardíaca.
Métodos: Muestras: En este estudio se analizaron 200 muestras correspondientes a AD, AMI, VS y TA en un grupo de 50 pacientes con cardiopatía coronaria de la Región de Antofagasta, todos residentes en la Región por a lo menos 5 años, (grupo expuesto, E) que fueron sometidos a cirugía cardíaca entre los años 2004 y 2005 en la Clínica Antofagasta. Como grupo control (no expuesto, $\mathrm{NE})$ se estudiaron 80 muestras de 20 pacientes sometidos a cirugía del mismo tipo en este mismo período en el Hospital Clínico de la Pontificia de la Universidad Católica de Chile en Santiago, provenientes de la zona central y sur del país.

La preparación de las muestras se realizó en la "sala limpia" del laboratorio, equipada con una campana de flujo laminar (Labconco, Purifer clase II), utilizando dispositivos inertes de plástico y titanio, mortero de ágata y material quirúrgicos de acero inoxidable. Después de la eliminación de residuos, los tejidos fueron enjuagados con agua desionizada, separados como muestras unitarias y almacenados a $-20^{\circ} \mathrm{C}$. Posteriormente, se determinó la $\mathrm{CT}^{17}$ de los elementos en estudio. Además, se realizó una encuesta a estos pacientes, en las cuales se consideraron factores relacionados con el trabajo desarrollado, hábitos, lugares de residencia y permanencia en la ciudad.

El protocolo para Seres Humanos donantes de muestras fue aprobado por el Comité de Bioética de la Universidad de Antofagasta y Clínica Antofagasta

Determinación de CT de V, Mn, Cr, Ni, Co, Cu, As, Se y B: La mineralización de los tejidos $\mathrm{CV}$ se realizó por vía húmeda. Se pesaron entre $0,5-1,0 \mathrm{~g}$ de muestra en reactores de teflón, se adicionó $1 \mathrm{~mL}$ de agua desionizada Milli-Q, $10 \mathrm{~mL}$ de ácido nítrico, 500 $\mu \mathrm{L}$ de ácido sulfúrico y $300 \mu \mathrm{L}$ de ácido perclórico de calidad Suprapur (Merck). Luego se llevó a un horno con un riguroso programa y control de temperatura externa entre $170-180^{\circ} \mathrm{C}$ por 2 horas.

La CT de As y Se en las muestras se determinó por Generación de Hidruros, en la que se utiliza ácido clorhídrico de calidad suprapur, (Merck), Hidróxido de Sodio (Merck) y Borohidruro Sódico (Aldrich).

Las mediciones de As y Se se realizaron en un Espectrofotómetro de Absorción Atómica modelo GBC 909 acoplado a un Generador de Hidruros GBC HG-3000, equipado con una manta electrotermal modelo GBC EHG - 3000. Como fuente de excitación se utilizó una lámpara de cátodo hueco BDL (Photron).

Las CT de V, Cr, Co, Ni y Cu se determinaron por Es- 
pectrometría de Absorción Atómica con Horno de Grafito (GFAAS) y las mediciones se llevaron a cabo en un Espectrofotómetro de Absorción Atómica modelo GBC 933A (Australia).

Las CT de Mn se determinaron por espectrometría de absorción atómica con llama y nebulización hidráulica de de alta presión (HHPN-FF-AAS).

La CT de B se determinó por Espectrometría de Emisión Atómica de Plasma Inductivamente Acoplado con Detección Óptica (ICP-OES) y las mediciones se llevaron a cabo en un equipo GBC Integra XL (Australia).

Estadística: El Análisis estadístico ${ }^{18}$ se realizó mediante el programa STATISTICA 6.1 (StatSoft, Tulsa, OK); El nivel alpha para significación estadística se fijó en p< 0,05 . Con el propósito de obtener información sobre las relaciones de similaridad interelementales en un espacio multidimensional, se aplicó el análisis de Cluster ${ }^{18}$, considerado para medir la distancia entre las variables, el método de Ward aplicando el criterio de 1-Pearson $\mathrm{r}$.

Las mediciones se validaron empleando Materiales de Referencia Certificados DORM-1, DORM-2,LUTS-1 y TORT-2 y mediante la recuperación de concentraciones adicionadas desde patrones primarios.

Resultados: La Tabla 1 muestra los niveles de $\mathrm{V}, \mathrm{Cr}$, $\mathrm{Mn}, \mathrm{Co}, \mathrm{Ni}, \mathrm{Cu}, \mathrm{B}, \mathrm{As}$ y Se en AD, VS, AMI y TA de un grupo de pacientes de la Región de Antofagasta, sometidos a cirugía cardíaca entre los años 2004-2005. Las CT se complementan con una encuesta demográfica aplicada a los pacientes.

En el grupo E el promedio de edad fue de 70,9 \pm 6,5 años En ellos, B presentó correlaciones interelementales significativas $(\mathrm{p}=0,05)$, que no se presentaron o fueron muy escasa en el grupo NE. En el grupo E el rango de CT de $\mathrm{B}$ en $\mathrm{AD}$ fue de 24,721 - 932,032 $\mu \mathrm{g} / \mathrm{g}$, niveles muy superiores a los encontrados en el resto de los elementos en estudio. La VSE presentó también contenidos altos de B $(54,759-1400,323 \mu \mathrm{g} / \mathrm{g})$. Niveles de MP inferiores y similares se encontraron para $\mathrm{Mn}$ y $\mathrm{Cu}$ en $\mathrm{AD}(0,4191$ $-8,317 \mu \mathrm{g} / \mathrm{g}), \mathrm{TA}(0,3954-6,476 \mu \mathrm{g} / \mathrm{g})$ y $\mathrm{AD}(0,0201$ $-1,2678 \mu \mathrm{g} / \mathrm{g})$ y AMI $(0,061-1,172 \mu \mathrm{g} / \mathrm{g})$, respectivamente.

En el grupo NE el promedio de edad fue de 60,2 \pm 9,6 años. El rango de $\mathrm{CT}$ de $\mathrm{B}$ encontrada en $\mathrm{AD}$ fue de $25,205-56,873 \mu \mathrm{g} / \mathrm{g}$ y en VS $23,25-56,67 \mu \mathrm{g} / \mathrm{g}$.

El orden decreciente de la carga de estos elementos en los

\begin{tabular}{|c|c|c|c|c|c|c|c|}
\hline ELEMENTO & MATRIZ (N) & PROM & EDIO & $\mathrm{E}( \pm)$ & MIN - MAX & MEDI & ANA \\
\hline V & $\begin{array}{l}\text { AD (50) } \\
\text { AMI (52) } \\
\text { VS (52) } \\
\text { TA (53) }\end{array}$ & $\begin{array}{l}0,48 \\
0,55 \\
0,78 \\
0,36\end{array}$ & & $\begin{array}{l}0,32 \\
0,33 \\
0,63 \\
0,40\end{array}$ & $\begin{array}{l}0,089-1,61 \\
0,11-2,21 \\
0,24-3,31 \\
0,057-1,09\end{array}$ & $\begin{array}{l}0,43 \\
0,50 \\
0,56 \\
0,30\end{array}$ & \\
\hline $\mathrm{Cr}$ & $\begin{array}{l}\text { AD (48) } \\
\text { AMI (52) } \\
\text { VS (51) } \\
\text { TA (53) }\end{array}$ & $\begin{array}{l}0,30 \\
0,40 \\
0,44 \\
0,37\end{array}$ & & $\begin{array}{l}0,29 \\
0,32 \\
0,33 \\
0,26\end{array}$ & $\begin{array}{l}0,028-1,64 \\
0,021-1,31 \\
0,043-1,36 \\
0,057-1,09\end{array}$ & $\begin{array}{l}0,22 \\
0,34 \\
0,35 \\
0,30\end{array}$ & \\
\hline Mn & $\begin{array}{l}\text { AD (50) } \\
\text { AMI (52) } \\
\text { VS (52) } \\
\text { TA (53) }\end{array}$ & $\begin{array}{l}2,60 \\
1,97 \\
4,75 \\
1,63\end{array}$ & $\begin{array}{l}5,33 \\
1,70 \\
4,04 \\
1,63\end{array}$ & $\begin{array}{l}1,73 \\
1,02 \\
4,18 \\
1,17\end{array}$ & $\begin{array}{l}0,42-8,32 \\
0,56-6,56 \\
1,43-23,0 \\
0,40-6,48\end{array}$ & $\begin{array}{l}2,50 \\
1,73 \\
3,76 \\
1,31\end{array}$ & $\begin{array}{l}3,67 \\
1,01 \\
2,50 \\
0,92\end{array}$ \\
\hline Co & $\begin{array}{l}\text { AD (50) } \\
\text { AMI (50) } \\
\text { VS (51) } \\
\text { TA (53) }\end{array}$ & $\begin{array}{l}0,45 \\
0,34 \\
0,62 \\
0,35\end{array}$ & & $\begin{array}{l}0,43 \\
0,20 \\
0,54 \\
0,37\end{array}$ & $\begin{array}{l}0,030-2,24 \\
0,029-1,16 \\
0,092-3,38 \\
0,070-2,06\end{array}$ & $\begin{array}{l}0,37 \\
0,31 \\
0,50 \\
0,23\end{array}$ & \\
\hline $\mathrm{Ni}$ & $\begin{array}{l}\text { AD (50) } \\
\text { AMI (51) } \\
\text { VS (52) } \\
\text { TA (53) }\end{array}$ & $\begin{array}{l}0,24 \\
0,29 \\
0,64 \\
0,26\end{array}$ & & $\begin{array}{l}0,18 \\
0,24 \\
0,74 \\
0,35\end{array}$ & $\begin{array}{l}0,039-1,02 \\
0,017-1,01 \\
0,066-4,70 \\
0,022-2,20\end{array}$ & $\begin{array}{l}0,20 \\
0,21 \\
0,48 \\
0,15\end{array}$ & \\
\hline $\mathrm{Cu}$ & $\begin{array}{l}\text { AD (50) } \\
\text { AMI (52) } \\
\text { VS (52) } \\
\text { TA (53) }\end{array}$ & $\begin{array}{l}0,31 \\
0,16 \\
0,72 \\
0,093\end{array}$ & $\begin{array}{l}5,49 \\
1,99 \\
6,03 \\
1,97\end{array}$ & $\begin{array}{l}0,28 \\
0,19 \\
0,93 \\
0,11\end{array}$ & $\begin{array}{l}0,020-1,29 \\
0,006-1,17 \\
0,021-4,59 \\
0,007-0,49\end{array}$ & $\begin{array}{l}0,20 \\
0,12 \\
0,36 \\
0,044\end{array}$ & $\begin{array}{l}3,79 \\
0,97 \\
3,02 \\
0,75\end{array}$ \\
\hline B & $\begin{array}{l}\text { AD (50) } \\
\text { AMI (52) } \\
\text { VS (52) } \\
\text { TA (53) }\end{array}$ & $\begin{array}{l}270,9 \\
128,5 \\
299,3 \\
155,9\end{array}$ & $\begin{array}{l}92.13 \\
24.32 \\
31.49 \\
28.07\end{array}$ & \begin{tabular}{|l|l|}
205,6 \\
90,0 \\
281,3 \\
153,1
\end{tabular} & $\begin{array}{l}24,7-932,0 \\
25,3-594,7 \\
54,8-1400,3 \\
25,7-915,8\end{array}$ & $\begin{array}{l}239,1 \\
105,0 \\
204,7 \\
120,2\end{array}$ & $\begin{array}{l}63.63 \\
33.00 \\
37.63 \\
35.97\end{array}$ \\
\hline As & $\begin{array}{l}\text { AD (53) } \\
\text { AMI (53) } \\
\text { VS (52) } \\
\text { TA (54) }\end{array}$ & $\begin{array}{l}0,56 \\
0,29 \\
0,61 \\
0,32\end{array}$ & $\begin{array}{l}4,85 \\
1,52 \\
2,97 \\
1,27\end{array}$ & $\begin{array}{l}0,47 \\
0,26 \\
0,40 \\
0,26\end{array}$ & $\begin{array}{l}0,087-2,39 \\
0,066-1,76 \\
0,22-2,16 \\
0,071-1,51\end{array}$ & $\begin{array}{l}0,47 \\
0,22 \\
0,47 \\
0,23\end{array}$ & $\begin{array}{l}3,79 \\
0,90 \\
2,13 \\
0,78\end{array}$ \\
\hline $\mathrm{Se}$ & $\begin{array}{l}\text { AD (53) } \\
\text { AMI (53) } \\
\text { VS (51) } \\
\text { TA (54) }\end{array}$ & $\begin{array}{l}0,60 \\
0,33 \\
0,64 \\
0,33\end{array}$ & $\begin{array}{l}1,88 \\
0,72 \\
1,29 \\
0,57\end{array}$ & $\begin{array}{l}0,47 \\
0,20 \\
0,45 \\
0,24\end{array}$ & $\begin{array}{l}0,091-2,49 \\
0,075-1,28 \\
0,12-2,30 \\
0,094-1,17\end{array}$ & $\begin{array}{l}0,49 \\
0,28 \\
0,50 \\
0,24\end{array}$ & $\begin{array}{l}1,44 \\
0,44 \\
0,85 \\
0,31\end{array}$ \\
\hline
\end{tabular}

Tabla 1. Carga de V, Cr, Mn, Co, Ni, Cu, B, As y Se en tejidos cardiovasculares de un grupo de pacientes Expuestos de la Región Antofagasta - Chile, sometidos a cirugía cardiaca entre los años 2004-2005. Concentraciones en $\mu \mathrm{g} / \mathrm{g}$ base seca. Se destaca en rojo pacientes sometidos a cirugía cardiaca entre los años 1995 -2000.

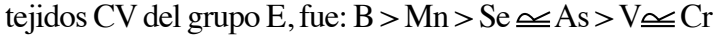
$\simeq \mathrm{Co}>\mathrm{Cu} \cong \mathrm{Ni}$, en que los niveles de concentración de As, $\mathrm{Se}, \mathrm{Mn}$ y Cu fueron más bajos que los medidos en trabajos anteriores en tejidos de pacientes operados entre 1995 - 2000, los cuales han sido destacados en rojo en las Tabla 1 y 2 . En la Tabla 2 se muestran los resultados en los tejidos del grupo control. 


\begin{tabular}{|c|c|c|c|c|c|c|c|}
\hline ELEMENTO & MATRIZ & PROMF & EIO & $\mathrm{DE}( \pm)$ & MIN - MAX & MEDI & ANA \\
\hline V & $\begin{array}{l}\text { AD } \\
\text { AMI } \\
\text { VS } \\
\text { TA }\end{array}$ & $\begin{array}{l}0,30 \\
0,16 \\
0,35 \\
0,14\end{array}$ & & \begin{tabular}{l|l|}
0,18 & \\
0,06 & \\
0,11 & \\
0,046 &
\end{tabular} & $\begin{array}{l}0,090-0,79 \\
0,075-0,27 \\
0,22-0,57 \\
0,076-0,24\end{array}$ & $\begin{array}{l}0,27 \\
0,14 \\
0,31 \\
0,12\end{array}$ & \\
\hline $\mathrm{Cr}$ & $\begin{array}{l}\text { AD } \\
\text { AMI } \\
\text { VS } \\
\text { TA }\end{array}$ & \begin{tabular}{l|l}
0,21 \\
0,14 \\
0,42 \\
0,14
\end{tabular} & & \begin{tabular}{l|l|}
0,18 & \\
0,076 & \\
0,25 & \\
0,066 &
\end{tabular} & $\begin{array}{l}0,031-0,87 \\
0,059-0,32 \\
0,14-0,91 \\
0,053-0,30\end{array}$ & $\begin{array}{l}0,19 \\
0,11 \\
0,35 \\
0,12\end{array}$ & \\
\hline $\mathrm{Mn}$ & $\begin{array}{l}\text { AD } \\
\text { AMI } \\
\text { VS } \\
\text { TA }\end{array}$ & \begin{tabular}{l|}
0,66 \\
0,34 \\
1,12 \\
0,22
\end{tabular} & $\begin{array}{l}2,65 \\
1,54 \\
5,26 \\
1,26\end{array}$ & \begin{tabular}{l|l|}
0,44 & \\
0,10 & \\
0,36 & \\
0,078 &
\end{tabular} & $\begin{array}{l}0,13-1,96 \\
0,16-0,65 \\
0,57-2,21 \\
0,086-0,34\end{array}$ & $\begin{array}{l}0,67 \\
0,32 \\
1,05 \\
0,23\end{array}$ & $\begin{array}{l}1,36 \\
0,77 \\
4,90 \\
1,11\end{array}$ \\
\hline Co & $\begin{array}{l}\text { AD } \\
\text { AMI } \\
\text { VS } \\
\text { TA }\end{array}$ & \begin{tabular}{l|}
0,34 \\
0,14 \\
0,35 \\
0,14
\end{tabular} & & \begin{tabular}{l|l|}
0,31 & \\
0,047 & \\
0,13 & \\
0,071 &
\end{tabular} & $\begin{array}{l}0,067-1,49 \\
0,085-0,24 \\
0,20-0,69 \\
0,067-0,30\end{array}$ & $\begin{array}{l}0,25 \\
0,13 \\
0,30 \\
0,12\end{array}$ & \\
\hline $\mathrm{Ni}$ & $\begin{array}{l}\text { AD } \\
\text { AMI } \\
\text { VS } \\
\text { TA }\end{array}$ & \begin{tabular}{l|l|}
0,12 \\
0,15 \\
0,36 \\
0,14
\end{tabular} & & \begin{tabular}{l|l}
0,065 \\
0,071 \\
0,25 \\
0,083
\end{tabular} & $\begin{array}{l}0,025-0,24 \\
0,010-0,26 \\
0,022-1,10 \\
0,016-0,37\end{array}$ & \begin{tabular}{l|l|}
0,097 & \\
0,16 \\
0,35 \\
0,13
\end{tabular} & \\
\hline $\mathrm{Cu}$ & $\begin{array}{l}\text { AD } \\
\text { AMI } \\
\text { VS } \\
\text { TA }\end{array}$ & $\begin{array}{l}0,093 \\
0,052 \\
0,24 \\
0,028\end{array}$ & $\begin{array}{l}3,83 \\
2,05 \\
6,94 \\
1,46\end{array}$ & \begin{tabular}{l|l|}
0,076 & \\
0,028 & \\
0,17 & \\
0,016 &
\end{tabular} & $\begin{array}{l}0,010-0,29 \\
0,009-0,12 \\
0,029-0,70 \\
0,008-0,053\end{array}$ & $\begin{array}{l}0,080 \\
0,052 \\
0,17 \\
0,025\end{array}$ & $\begin{array}{l}2,88 \\
0,93 \\
3,23 \\
0,94\end{array}$ \\
\hline B & $\begin{array}{l}\text { AD } \\
\text { AMI } \\
\text { VS } \\
\text { TA }\end{array}$ & \begin{tabular}{l|}
24.1 \\
10,3 \\
33,1 \\
9,22
\end{tabular} & & $\begin{array}{l}8,25 \\
2,38 \\
8,25 \\
2,89\end{array}$ & $\begin{array}{l}25,2-56,9 \\
5,45-13,6 \\
25,2-56,9 \\
3,75-14,7\end{array}$ & $\begin{array}{l}30,0 \\
10,7 \\
30,0 \\
8,24\end{array}$ & \\
\hline As & $\begin{array}{l}\text { AD } \\
\text { AMI } \\
\text { VS } \\
\text { TA }\end{array}$ & \begin{tabular}{l|l|}
0,32 \\
0,14 \\
0,28 \\
0,15
\end{tabular} & $\begin{array}{l}2,56 \\
0,58 \\
3,79 \\
0,72\end{array}$ & \begin{tabular}{|l|l|l|}
0,17 & \\
0,040 & \\
0,084 & \\
0,059 &
\end{tabular} & $\begin{array}{l}0,12-0,72 \\
0,076-0,20 \\
0,17-0,44 \\
0,073-0,28\end{array}$ & $\begin{array}{l}0,31 \\
0,13 \\
0,30 \\
0,13\end{array}$ & $\begin{array}{l}1,62 \\
0,51 \\
1,99 \\
0,51\end{array}$ \\
\hline $\mathrm{Se}$ & $\begin{array}{l}\text { AD } \\
\text { AMI } \\
\text { VS } \\
\text { TA }\end{array}$ & \begin{tabular}{l|}
0,91 \\
0,31 \\
0,79 \\
0,42
\end{tabular} & $\begin{array}{l}1,09 \\
0,41 \\
1,84 \\
0,31\end{array}$ & $\begin{array}{l}0,61 \\
0,14 \\
0,56 \\
0,33\end{array}$ & $\begin{array}{l}0,21-2,43 \\
0,16-0,75 \\
0,26-2,10 \\
0,14-1,27\end{array}$ & $\begin{array}{l}0,75 \\
0,26 \\
0,62 \\
0,29\end{array}$ & $\begin{array}{l}0,91 \\
0,43 \\
1,15 \\
0,27\end{array}$ \\
\hline
\end{tabular}

Tabla 2. Carga de V, Cr, Mn, Co, $\mathrm{Ni}, \mathrm{Cu}, \mathrm{B}$, As y Se en tejidos cardiovasculares de un grupo de 20 pacientes No Expuestos de Regiones del Centro y Sur de Chile, sometidos a cirugía cardiaca entre los años 2004-2005. Concentraciones en $\mu \mathrm{g} / \mathrm{g}$ base seca. Se destaca en rojo pacientes sometidos a cirugía cardiaca entre los años 1995 - 2000.

Las relaciones inter elementales de similaridad multivariante en $\mathrm{AD}$ del grupo $\mathrm{E}$ y del grupo control $\mathrm{NE}$ se presentan en los Dendrogramas de la Figuras 1. En los pacientes E (Figura 1a) se observan dos grupos claramente diferenciados, existe una relación interelemental directa entre $\mathrm{Cu}-\mathrm{Ni}$, Se - Co, B - V. Mientras que Cr -As y Mn tienen un comportamiento independiente en el tejido. En el grupo control NE (Figura 1b) se observa una relación interelemental directa entre $\mathrm{Co}-\mathrm{Cr}$, As - Mn y Ni - V. A diferencia del grupo $\mathrm{E}$ actúan independientemente $\mathrm{B}-\mathrm{Se}$ y $\mathrm{Cu}$.
Figura 1.

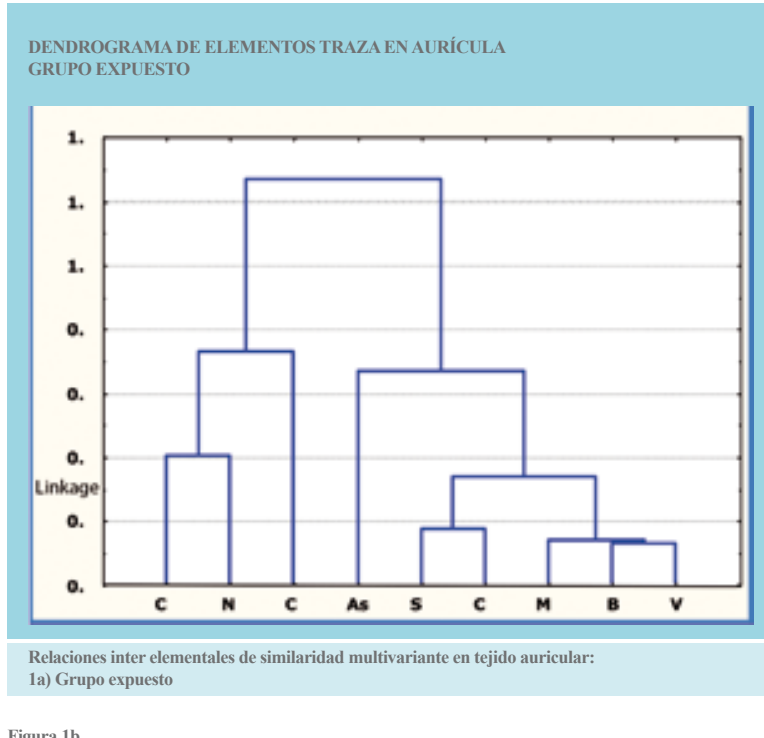

Figura 1b.

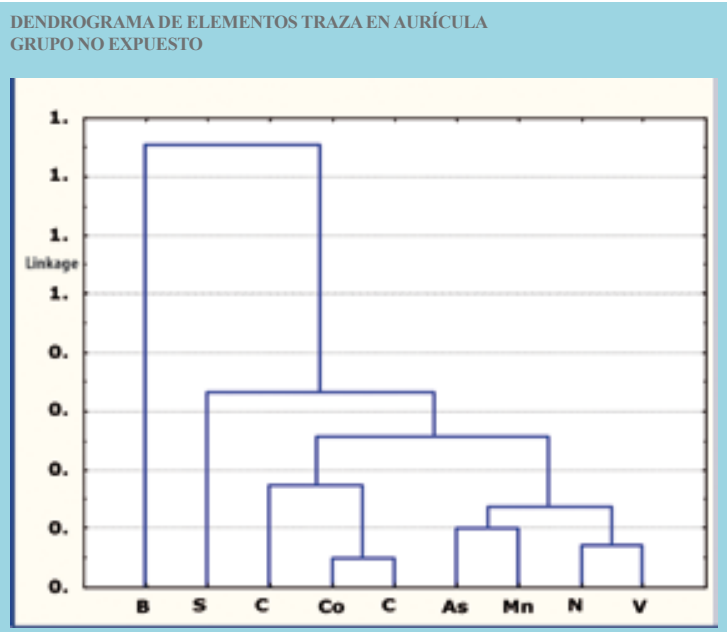

Figura 1b) Relaciones inter elementales de similaridad multivariante en el tejido auricular del Grupo No Expuesto.

Discusión: Los elementos V, Cr, Mn, Co, Ni, Cu, As y Se son considerados importantes para la salud cardiovascular. En este estudio, se incluyó entre los elementos investigados también a $\mathrm{B}$, debido al enriquecimiento que este elemento presenta en las aguas del río Loa. Puede inferirse que los tejidos CV de pacientes de la Región de Antofagasta reflejan la huella digital de la fuente de agua de consumo humano, intervenida para bajar los niveles de As en plantas de abatimiento. Se considera que los factores demográficos son menos importantes que los factores transversales como calidad del agua, aire y alimentación. La metalogenia de la Región de Antofagasta que determina su gran actividad minera y la dureza de sus aguas 
continentales, de origen predominantemente sub superficial, enriquecidas en B, As y otros metales, la convierten en un interesante caso de estudio en relación con cierto tipo de enfermedades de alta incidencia, como las patologías cardiovasculares. De acuerdo a nuestros resultados, la presencia de las altas concentraciones de ET y MP en los tejidos expuestos podría indicar que las poblaciones que consumen aguas muy duras presenten un alto riesgo de patologías cardiovasculares y cerebrovasculares.

Individualmente el elemento más enriquecido en todos los tipos de tejidos del grupo E de la Región de Antofagasta es B, le siguen As y Mn. Los ordenes de enriquecimiento por tejido del grupo de pacientes $\mathrm{E}$ fueron, $\mathrm{AD}$ : $\mathrm{B}$ $>\mathrm{Mn}>\mathrm{Se} \equiv \mathrm{As}>\mathrm{V} \equiv \mathrm{Co}>\mathrm{Cu} \equiv \mathrm{Cr}>\mathrm{Ni}$; AMI: $\mathrm{B}>>$ $\mathrm{Mn}>\mathrm{V}>\mathrm{Co}>\mathrm{Cr}>\mathrm{Se} \equiv \mathrm{Ni} \equiv \mathrm{As}>\mathrm{Cu}$. En el grupo NE $\mathrm{Cu}$ está empobrecido, en particular en AD y AMI.

El notable enriquecimiento de los elementos en los tejidos CV del grupo expuesto, señala que estos tejidos son buenos bioindicadores de la exposición a ET y MP in- volucrados en el riesgo cardiovascular. La aplicación de la técnica de cluster permitió demostrar que los agrupamientos interelementales son diferentes para un mismo tipo de tejido, tanto en el grupo de pacientes de la Región de Antofagasta E como en el grupo control NE, lo cual permite inferir que las interrelaciones elementales podría ser también diferentes. Estas relaciones pueden afectar de manera también diferente a la capacidad antioxidante de los tejidos, inhibiendo la superóxido dismutasa. La mayor carga de ET y MP en los tejidos del grupo expuesto favorecería el estrés oxidativo y la mineralización de los tejidos CV.

Agradecimientos: Se agradece el financiamiento de esta Investigación al Proyecto DI UA código S/F - 005 - 06 y Asistencia Técnica Universidad de Antofagasta Chile. El aporte de los tejidos Cardiovasculares en estudio a Clínica Antofagasta - Chile y Hospital Clínico de la Pontificia Universidad Católica de Chile.

\section{Referencias:}

1. TAKÁCS S, TATAR A. Trace elements in the environment and in human organs. Environmental Research. 1987; 42: $312-20$

2. FRIEDEN E. New perspertives on the essential trace elements. J. Chem. Educ.1985; 62: 917 - 23.

3. SELINUS O, ALLOWAY B, CENTENO JA, FINKElMAN RB, FUGE R, SMEDLEY VP. Essential of Medical Geology. Impacts of the Natural Environmental on Public Health. USA, London Elsevier, 2005.
4. ROMÁN-SILVA DA, RIVERA L, MORALES T, ÁVILA J, CORTÉS P. Determination of trace elements in environmental and biological samples using improved sample introduction in flame atomic absorption spectrometry (HHPN - AAS; HHPN - FF- AAS). Intern J Environ Anal Chem. 2003; 83: 327 - 41 .

5. NG JC. Environmental contamination of arsenic and its toxicological impact on humans. Environ Chem. 2005; $2: 146-160$.

6. ALTEKIN E, COKER C, SISMAN AR, ÔNVURAL B, KURALAY F, KIRIMILI Ô. The relationship between trace elements and cardiac markers in acute coronary syndromes. J Trace Elem Med Biol. 2005; 18: 235 - 42. 
7. ANDREW A, BURGUESS JL, MEZA MM, DEMIDENKO E, WAUGH MG, HAMILTON JW, et al. Arsenic exposure is associated with decreased DNA repair in vitro and in individuals exposed to drinking water arsenic. Environ Health Perspect. 2006; 18: 1193 - 98.

8. TANGUY S, TOUFEKTSIAN MC, BESSE S, DUCROS V, DE LEIRIS J, BOUCHER F. Dietary selenium intake affects cardiac susceptibility to ischemia / reperfusion in male senescent rats. Age and Ageing. 2003; 32: 273 - 78 .

9. STRAIN JJ. Trace elements and cardiovascular disease. In: Sandström B., Walter P., editors, Role of trace elements for health promotion and disease prevention. Nutr Dieta Basel Karget, 1998; 54: 127 - 140.

10. NSCHRAUZER G. Anticarcinogenic effects of selenium. Cellular and Molecular. Life Sciences. 2000; 57:1864 - 73.

11. TINGGI U. Selenium: its role as antioxidant in human health. Environ Health Prev Med. 2008; 13:102-8.

12. SAXENA R, JAISWA LG. Selenium and its role in health and disease. Kuwait Medical Journal. 2007; 39: 10-18.

13. BARANDIER C, TANGUY S, PUCHEU S, BOUCHIER
F, DE LEIRIS. J. Effect of antioxidant trace elements on the response of cardiac tissue to oxidative stress. Ann N Y Acad Sci. 1999; 874: 138 - 55.

14. ZACHARA B, SALAK A, KOTERSKA D, MANITIUS J, WASOWICZ W. Selenium and glutathione peroxidases in blood of patients with different stages of chronic renal failure. J. Trace Elem. Med Biol. 2004; 17:291- 99.

15. ROMÁN DA, PIZARRO I, RIVERA L, CÁMARA C, PALACIOS MA, GÓMEZ MM, et al. An approach to the arsenic status in cardiovascular tissues of patients with coronary heart disease. Hum Exp Toxicol. 2010; 30: 1150 -64 .

16. COUGHLIN J. Sources of human exposure. Overview of water supplies as sources of boron. Biol Trace Element Res. 1998; 66: 87-100.

17. SOLAR C, PIZARRO I, ROMAN D. Presencia de altos niveles de arsénico en tejidos cardiovasculares de pacientes de áreas contaminadas de Chile. Revista Chilena de Cardiología. 2012; 30: 221-27.

18. FORINA M, ARMANINO C, RAGGIO V. Clustering with dendrograms on interpretation variables. Anal Chim Acta. 2002; 454:13 - 19. 